\title{
Thermal Infrared Hot Spot and Dependence on Canopy Geometry
}

\author{
James A. Smith, FELLOW SPIE \\ NASA Goddard Space Flight Center \\ Laboratory for Terrestrial Physics \\ Greenbelt, MD 20771 \\ Telephone: (301) 614-6020 \\ FAX: (301) 614-6015 \\ E-mail: jasmith@hemlock.gsfc.nasa.gov
}

Jerrell R. Ballard, Jr., MEMBER SPIE

USAE Research and Development Center

Environmental Laboratory

Vicksburg, MS 39180

Telephone: (601) 634-2936

FAX: (601) 634-3726

E-mail: ballardj@wes.army.mil 


\begin{abstract}
We perform theoretical calculations of the canopy thermal infrared (TIR) hot spot using a first principles 3-D model described earlier. Various theoretical canopies of varying leaf size and for differing canopy height are used to illustrate the magnitude of the TIR effect. Our results are similar to predicted behavior in the reflective hot spot as a function of canopy geometry and comparable to TIR measurements from the literature and our own simple ground experiments. We apply the MODTRAN atmospheric code to estimate the at-sensor variation in brightness temperature with view direction in the solar principal plane. For simple homogeneous canopies, we predict canopy thermal infrared hot spot variations of 2 degrees $C$ at the surface with respect to nadir viewing. Dependence on leaf size is weak as long as the ratio of leaf size to canopy height is maintained. However, the angular width of the hot spot increases as the ratio of leaf diameter to canopy height increases. Atmospheric effects minimize but do not eliminate the TIR hot spot at satellite altitudes.
\end{abstract}

\title{
Subject terms:
}

remote sensing; thermal infrared; brightness temperature; directional anisotropy, hot spot; canopy model; canopy geometry 


\section{Introduction}

In this paper, we look at a new, relatively unexplored remote sensing aspect: the canopy thermal infrared hot spot. A maximum in thermal infrared exitance occurs when the viewer is positioned in the anti-solar direction along the principal plane and observes warmer sunlit surfaces. A similar phenomenon is easily observed in the reflective regime but arises from a lack of visible shadows in the anti-solar direction. It has been extensively studied theoretically at these shorter wavelengths ${ }^{1}$. There are experimental reports of thermal infrared hot spot effects from both ground and aircraft platforms. Balick and Hutchinson ${ }^{2}$ describe tower results from a leafless deciduous canopy exhibiting strong thermal differences between the canopy over and under story and the litter background. Strong asymmetric heating of tree trunks was evident with angular variations of 3 to $5 \mathrm{deg}$ C. Lagouarde, et al. ${ }^{3}$ report hot spot variations in the solar principal of 2 $\operatorname{deg} \mathrm{C}$ for a pine canopy as measured from an aircraft platform.

Interestingly, the authors have not found theoretical analyses of the thermal infrared hot spot in the reviewed literature or, at least details describing the dependence of the TIR hot spot on canopy geometry. With increasing capabilities in military and civilian thermal infrared remote sensing systems, accounting for TIR hot spot variations would appear to be important in representing thermal infrared background clutter. Synthetic scene generation models ${ }^{4}$ can address these needs, and such models are used to evaluate the performance of automatic target recognition algorithms ${ }^{5}$. Nevertheless, simulating realistic backgrounds remains one of the most difficult tasks for synthetic scene generation.

Environmental sensing also requires accurate estimates of satellite-derived surface temperature. This is now becoming feasible with new high resolution and high fidelity systems such as the Department of Energy Multispectral Thermal Imager ${ }^{6}$. This system offers several multispectral thermal infrared channels at $20 \mathrm{~m}$ spatial resolution.

We perform theoretical calculations of the canopy TIR hot spot in the solar principal plane for vegetation canopies using a three-dimensional thermal infrared exitance model we previously have reported and compared with measurements ${ }^{7}$. We estimate additional uncertainties induced 
by atmospheric directional variations using MODTRAN ${ }^{8}$. Our results are comparable to those reported in the literature and consistent with measurements we made for a simple grass canopy.

\section{Method}

Our approach to simulating canopy thermal infrared exitance has been described earlier? Basically, we first construct a three-dimensional representation of the canopy consisting of statistical ensembles of leaf surfaces distributed throughout the canopy volume and then raytrace the canopy to determine all of the elements in the scene that either are directly illuminated or in shade. We solve resulting energy budget equations to determine corresponding leaf temperatures for all sunlit and shaded leaf surfaces within the canopy. Finally, ray tracing is once again applied to project thermal exitance into the sensor field of view. Monte Carlo sampling is employed to reduce ray tracing times.

Here, we simulated a fairly continuous canopy $20 \mathrm{~m}$ by $20 \mathrm{~m}$ on a side with a Leaf Area Index of 3.0. For our nominal case, leaf facet size was taken to be $5 \mathrm{~cm}$ by $5 \mathrm{~cm}$ and the canopy 0.8 meters tall. The test scene contained 388,800 leaf facets randomly selected from a spherical leaf angle distribution. We selected a viewpoint 10 meters above the canopy. Given this geometry, the total field of view of the canopy was 70 degrees, i. e. plus or minus 35 degrees from zenith. Subsequently, leaf size also was taken to be $2.5 \mathrm{~cm}$ by $2.5 \mathrm{~cm}$. In addition, a canopy 1.6 meter in height was simulated. Figure la shows a hemispherical view of the simulated canopy with the viewer positioned at ground level and looking upwards towards the zenith.

We selected meteorological conditions corresponding to late morning, summer conditions at mid latitude. Solar zenith angle was 24 degrees, solar azimuth was 146 degrees, measured clockwise from North. Wind speed was $4 \mathrm{~m} \mathrm{~s}^{-1}$, relative humidity $55 \%$, air temperature $27.5^{\circ} \mathrm{C}$, and shortwave solar insulation $915 \mathrm{~W} \mathrm{~m}^{-2}$. Calculated sunlit temperatures ranged from 24 to $32 \mathrm{deg} \mathrm{C}$ with warmer temperatures occurring more frequently. Shaded leaf temperature was $26.5 \mathrm{deg}$ C.

Subsequently, we positioned a viewer $10 \mathrm{~m}$ above the canopy and projected 70,000 rays towards the canopy along the solar principal plane. The rays were distributed plus or minus 35 degrees from zenith in equal angle increments, $.001 \mathrm{deg}$. For each ray we computed the thermal infrared 
exitance projected into the field-of-view. We then averaged the data to one degree bins by summing the contributions from 1000 rays within each one degree bin. We repeated our analyses for three different sampling transects aligned along the solar principal plane but of slightly different azimuths. Finally, in order to further reduce small-scale variations in the ray-traced results, we subsequently applied a moving average filter to the data with 5 deg zenith angle bin widths. Following Lagouarde, et al. ${ }^{3}$, we converted the data to apparent brightness temperature and plot the differences between off-nadir and nadir directions.

Fig. $1 \mathrm{~b}$ shows a visual simulation of the scene using a hemispherical projection out to 35 degrees and the viewer positioned $10 \mathrm{~m}$ above the canopy. The solar principal plane lies along the diagonal line with nadir indicated by the center dot. The solar reflective hot spot can be seen in the upper left position along the principal plane. Sun position is in the lower right.

\section{Simulation Results}

Figure 2a shows the results of our calculations for the differences in brightness temperature for our nominal canopy viewed from oblique versus nadir directions within the solar principal plane. The mean plus and minus the standard deviation curves computed from sampling along the three transects are plotted. While the curves contain sampling noise, the canopy hot spot effect is clearly evident. We obtain over a 2 deg $\mathrm{C}$ peak corresponding to the zenith view angle 24 degrees in the anti-solar direction. The width of the canopy hot spot is significant, and our results mirror similar curves given in Lagouarde, et al. ${ }^{3}$.

As a simple reality check, we also performed an experiment over an unmowed lawn canopy using an Omega Scope 2000A (8-14 micrometer) thermal infrared sensor. Solar zenith angle was 45 degrees. Measurements in the solar principal plane indicated a canopy thermal hot spot of nearly $4 \mathrm{deg} C$ variation between the solar and anti-solar azimuths. While the canopy was

fairly dense, some warmer soil surfaces were visible than in our theoretical canopies. Our main purpose in the experiment was to confirm the existence of the TIR hot spot even for dense vegetation canopies. 
In the reflective regime, the canopy reflectance hot spot has been shown to depend on canopy geometry $^{1}$. The magnitude and the angular width of the reflective hot spot is the same for canopies with differing leaf size and canopy height as long as the cover fraction and the ratio of leaf size to canopy height remains constant. Figure $2 b$ shows our results for the TIR hot spot corresponding to two different theoretical canopies of differing leaf size and canopy height but that also maintain a constant leaf size to canopy height ratio. Our curves also show the relative insensitivity of the hot spot magnitude and angular width in this case. However, in the reflective regime, the angular width of the reflective hot spot increases as the leaf size to canopy height ratio increases. Figure $2 \mathrm{c}$ presents our results for two theoretical canopies of the same height but differing leaf size. We also show an increase in the angular spread of the hot spot with increasing leaf size to canopy height ratio. In our case, we further show an increase in the magnitude of the TIR hot spot that is somewhat unexpected and needs to be explored further.

The atmosphere affects measurement of surface directional brightness temperature because of the differing optical path lengths through the atmosphere while observing the surface at different view angles. The TIR hot spot is clearly evident at the surface for our simulated canopy. In order to illustrate atmospheric effects on the TIR hot spot, we used the MODTRAN atmospheric code to compute the thermal infrared exitance at satellite altitude corresponding to the Landsat Thematic Mapper band 6, i.e. 10.4 to 12.5 micrometers. Atmospheric effects depend upon both surface temperature and zenith view direction. Using MODTRAN, we computed the contribution of the atmosphere to TIR radiance for selected discrete surface temperatures and zenith view directions encompassing the range of values observed in our theoretical canopies. We then used two-dimensional interpolation and a table look up scheme to retrieve brightness temperatures, inverted from the Planck function, for the observed surface temperatures and zenith view directions.

For zenith view angles up to 25 degrees atmospheric effects induce an additional uncertainty of $0.5 \mathrm{deg}$ C. Contributions of the atmosphere increase with increasing surface temperature and zenith view direction. Figure $2 \mathrm{~d}$ compares the TIR directional anisotropy in the solar principal plane compared to nadir calculated at the surface and at satellite altitude. While subdued, the TIR hot spot is still clearly discernable. 


\section{Summary}

We have illustrated the use of a three-dimensional canopy thermal infrared exitance model to compute the at-surface canopy thermal infrared hot spot variation and its dependence on simple canopy geometry. We found that dependence on leaf size is weak as long as the ratio of leaf size to canopy height is maintained. However, the angular width of the hot spot increases as the ratio of leaf diameter to canopy height increases. Atmospheric effects subdue, but do not eliminate, the TIR hot spot.

Only illustrative calculations are given in this short note. A potential limitation in the present study was our omission of multiple scattering for the thermal infrared flux ${ }^{9}$. We expect this effect to be small for the cases simulated because our scene contained only very high emissivity surfaces. Our ray tracing code accommodates multiple scattering but requires significantly more processing time. A further limitation in our study is that all of our surface elements had negligible heat capacity. Thermal inertia effects were, therefore, not addressed. We also believe it would be interesting to compute and compare the characteristics of the reflective and thermal infrared hot spot for the same canopies.

\section{Acknowledgements}

Support was provided by the NASA Earth Observing-1 Mission Instrument Performance Evaluation and Data Validation Program and, in part, by the U.S. Army Engineer Research and Development Center, Modeling and Simulation initiative. 


\section{References}

1. David L. B. Jupp and Alan H. Strahler, "A hotspot model for leaf canopies," Remote Sens. Environ. 38, 193-210 (1991)

2. Lee K. Balick and Boyd A. Hutchinson, "Directional thermal infrared exitance distributions from a leafless deciduous forest," IEEE Trans. Geosci. Remote Sensing 24, 453-458 (1986).

3. Jean-Pierre Lagouarde, Herve Ballans, Patrick Moreau, Dominique Guyon, and Damien Coraboeuf, "Experimental study of the brightness surface temperature angular variations of maritime pine (Pinus pinaster) stands," Remote Sens. Environ. 72, 17-34 (2000).

4. J. R. Schott, R. Raqueno, and C. Salvaggio, "Incorporation of time-dependent thermodynamic model and a radiation progragation model into infrared three-dimensional synthetic image generation," Opt. Eng. 31, 1505-1516 (1992)

5. Clarence P. Walters, Carl W. Hoover, Jr., and James A. Ratches, "Performance of an automatic target recognizer algorithm against real and two versions of synthetic imagery," Opt. Eng. 39, 2279-2284 (2000).

6. Paul G. Weber, Brian C. Brock, Alfred J. Garrett, Barham W. Smith, Christoph C. Borel, William B. Clodius, Steven C. Bender, R.Rex Kay, Max L. Decker, "Multispectral Thermal Imager Mission Overview," Proc. SPIE 3753, 340-346 (1999)

7. James A. Smith and Jerrell R. Ballard, Jr., "Effect of spatial resolution on thermal and nearinfrared sensing of canopies," Opt. Eng. 38, 1413-1423 (1999).

8. A. Berk, G. P. Anderson, P. K. Acharya, J. H. Chetwynd, L. S. Bernstein, E. P. Shettle, M. W. Matthew, and S. M. Adler-Golden, MODTRAN4 User's Manual, Air Force Research Laboratory, Space Vehicles Directorate, Hanscom AFB, Ma 01731-3010, 1999.

9. Xiaowen Li, Alan H. Strahler, and Mark A. Friedl, "A conceptual model for effective directional emissivity from nonisothermal surfaces," IEEE Trans. Geosci. Remote Sensing 37, 2508-2517 (1999). 


\section{List of Figures}

Figure 1a. Hemispherical view of simulated canopy with viewer positioned at ground level and looking upwards towards the zenith.

Figure $1 \mathrm{~b}$. Hemispherical view of simulated canopy with viewer positioned $10 \mathrm{~m}$ above the canopy. The solar principal plane is indicated by the diagonal line and nadir direction by the center dot. Total field of view is \pm 35 degrees.

Figure 2a. Plot of the TIR directional anisotropy in brightness temperature in the solar principal plane compared to nadir. The mean and plus and minus the standard deviation curves computed from three transects, each consisting of 70,000 rays sampled along the solar principal plane, are shown.

Figure 2b. Calculation of the TIR brightness temperature as a function of zenith view angle for two theoretical canopies of differing leaf size and canopy heights but identical leaf size to canopy height ratio. Curves show the relative insensitivity of the hot spot magnitude and width if the leaf size to canopy height ratio is preserved.

Figure 2c. Calculation of the TIR brightness temperature as a function of zenith view angle for two theoretical canopies of same height but differing leaf size. Curves show the increase in angular spread of the hot spot with increasing leaf size to canopy height ratio.

Figure 2d. Comparison of TIR directional anisotropy in the solar principal plane compared to nadir calculated at surface and at satellite altitude. Satellite curve corresponds to the Landsat 7 TIR band pass and is computed from MODTRAN derived table look-up tables. 


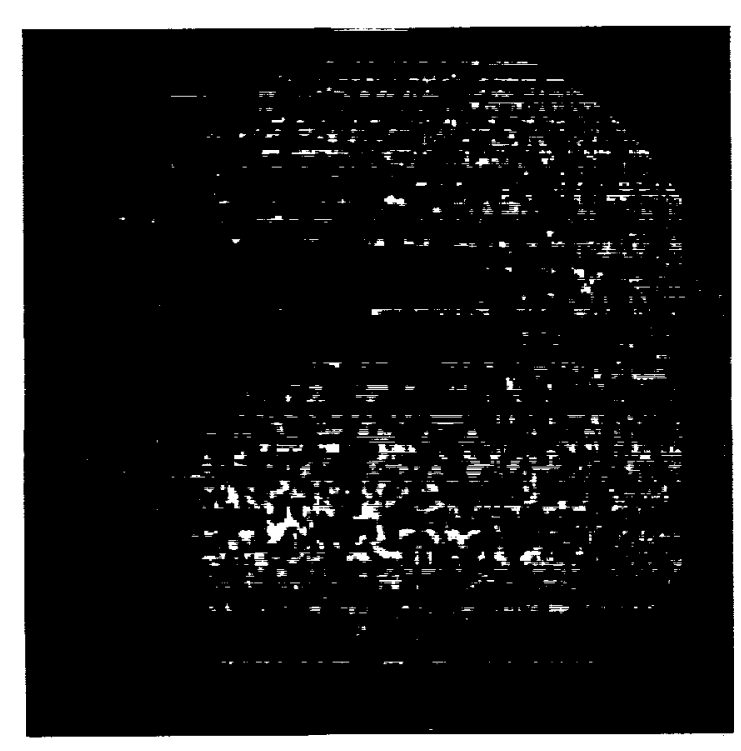

Figure 1a

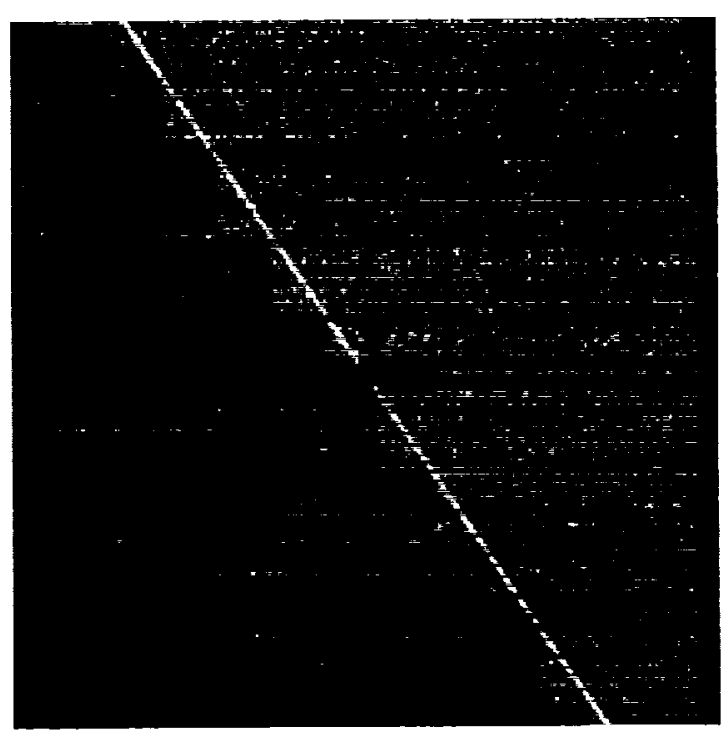

Figure 1b 


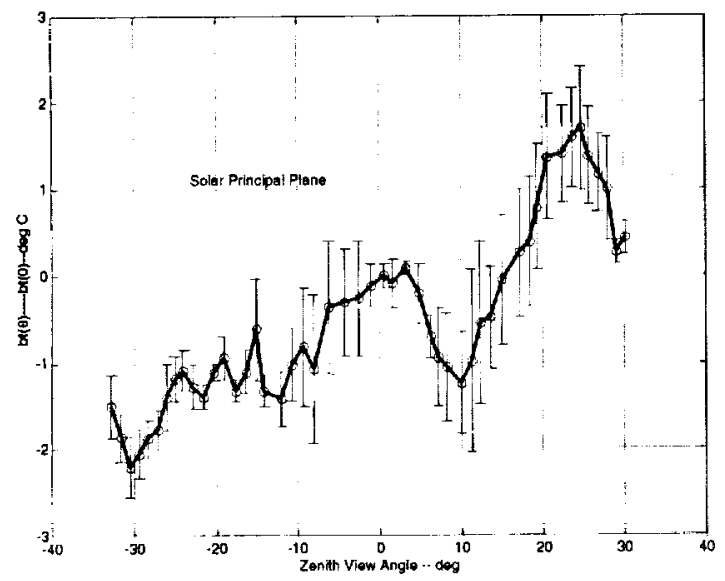

Figure 2a

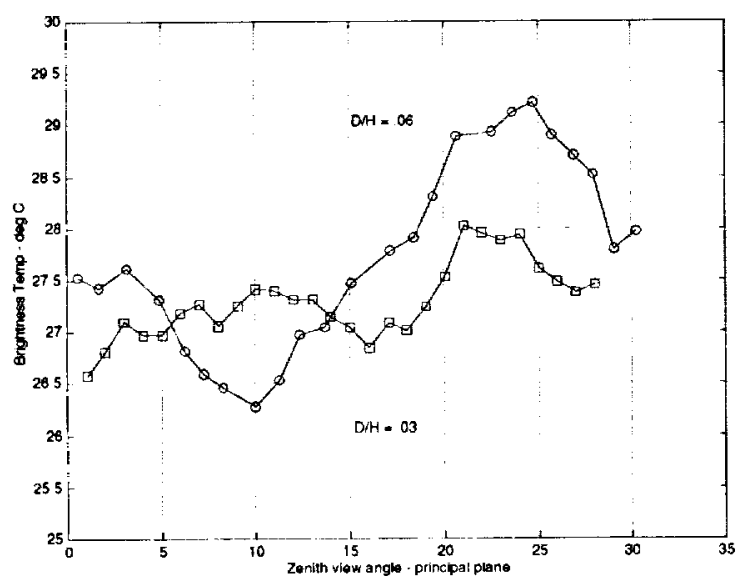

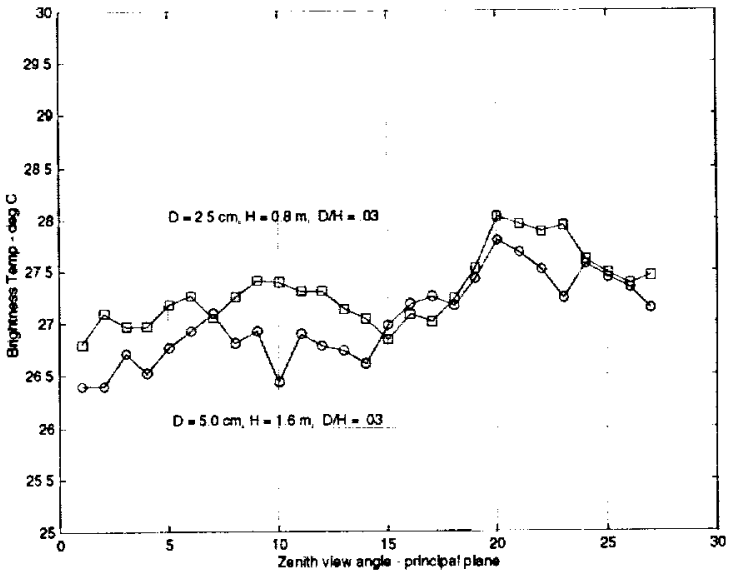

Figure 2b

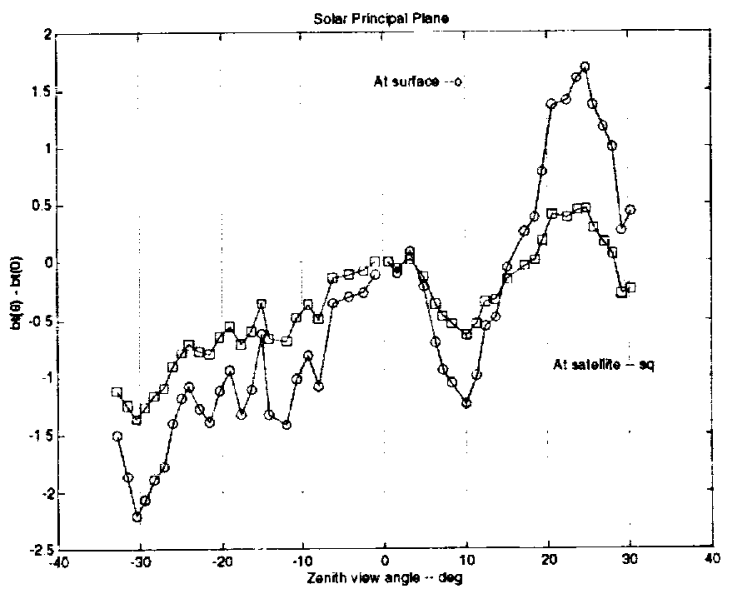

Figure 2d

Figure 2c 